\title{
Stieltjes moment problem in general Gelfand-Shilov spaces
}

by

\author{
Alberto Lastra and Javier Sanz (Valladolid)
}

\begin{abstract}
The Stieltjes moment problem is studied in the framework of general Gelfand-Shilov spaces, subspaces of the space of rapidly decreasing smooth complex functions, which are defined by imposing suitable bounds on their elements in terms of a given sequence $\boldsymbol{M}$. Necessary and sufficient conditions on $\boldsymbol{M}$ are stated for the problem to have a solution, sometimes coming with linear continuous right inverses of the moment map, sending a function to the sequence of its moments. On the way, some results on the existence of continuous right inverses for the Borel map are obtained for ultraholomorphic classes in sectors.
\end{abstract}

1. Introduction. The moment problem, with its many variations and generalizations, has inspired the work of mathematicians for over a century. Moments have been shown to be relevant in diverse areas of classical analysis, such as quadrature formulae, continued fractions, representation theory or spectral theory of operators, to name but a few. The book of N. I. Akhiezer [1] is a standard reference in this respect. Some more subjects, theoretical and applied, illuminated by this problem are described in [11], including questions in Fourier analysis, signal processing, inverse problems or statistics. Also, recent achievements relate this matter to the asymptotic expansion of generalized functions and the theory of distributional solutions of singular differential equations, as can be found in the book of R. Estrada and R. P. Kanwal [7] and the references therein.

In 1989 A. J. Durán [5] obtained the following answer to the so-called Stieltjes moment problem: there exists a function $f$ in the Schwartz space $\mathcal{S}(\mathbb{R})$ of rapidly decreasing smooth complex functions, with support in $[0, \infty)$ (we write $f \in \mathcal{S}(0, \infty)$ ) and with arbitrarily prescribed moments $\left\{\mu_{n}\right\}_{n=0}^{\infty}$, i.e., such that

$$
\int_{0}^{\infty} x^{n} f(x) d x=\mu_{n}, \quad n \in \mathbb{N}_{0}=\{0,1,2, \ldots\} .
$$

2000 Mathematics Subject Classification: Primary 47A57; Secondary 30E05, 44A60. Key words and phrases: Stieltjes moment problem, Gelfand-Shilov spaces, ultraholomorphic classes. 
The integral is called the $n$th moment of $f, \mu_{n}(f)$. Regarding subspaces of $\mathcal{S}(0, \infty)$, let us consider the so-called (classical) Gelfand-Shilov spaces $\mathcal{S}_{\alpha}(0, \infty)$, with $\alpha>0$, consisting of the functions $f \in \mathcal{S}(0, \infty)$ for which there exists $q=q(f)>0$ and, for any $m \in \mathbb{N}_{0}$, there exists $C_{m}=C_{m}(f)>0$ such that

$$
\sup _{x \in \mathbb{R}}\left|x^{n} f^{(m)}(x)\right| \leq C_{m} q^{n} n !^{\alpha}, \quad n \in \mathbb{N}_{0} .
$$

It is easy to see that whenever $f \in \mathcal{S}_{\alpha}(0, \infty)$, there exist $\tilde{C}_{0}, q_{0}>0$ such that

$$
\left|\mu_{n}(f)\right| \leq \tilde{C}_{0} q_{0}^{n} n !^{\alpha}, \quad n \in \mathbb{N}_{0},
$$

i.e., the sequence of moments is a Gevrey sequence (we write $\left\{\mu_{n}(f)\right\}_{n \in \mathbb{N}_{0}}$ $\left.\in \Lambda_{\alpha}\right)$. As a converse statement, relying heavily on an idea of A. L. Durán and R. Estrada [6] (who reproved A. J. Durán's result by the combination of Fourier transform techniques with the Borel-Ritt theorem), S.-Y. Chung, D. Kim and Y. Yeom obtained the following result.

TheOREM 1.1 ([4, Theorem 3.1]). Let $\alpha>2$, then for every sequence $\left\{\mu_{n}\right\}_{n \in \mathbb{N}_{0}} \in \Lambda_{\alpha}$ there exists $f \in \mathcal{S}_{\alpha}(0, \infty)$ with $\mu_{n}(f)=\mu_{n}, n \in \mathbb{N}_{0}$.

One may also consider the Gelfand-Shilov space $\mathcal{S}^{\alpha}(0, \infty)$, with $\alpha>0$, consisting of the functions $f \in \mathcal{S}(0, \infty)$ for which there exists $q=q(f)>0$ and, for any $m \in \mathbb{N}_{0}$, there exists $C_{m}=C_{m}(f)>0$ such that

$$
\sup _{x \in \mathbb{R}}\left|x^{m} f^{(n)}(x)\right| \leq C_{m} q^{n} n !^{\alpha}, \quad n \in \mathbb{N}_{0} .
$$

We note that, if $\alpha \leq 1$, these functions are analytic in a strip, and so $\mathcal{S}^{\alpha}(0, \infty)=\{0\}$.

With similar arguments to the ones employed for the previous theorem, J. Chung, S.-Y. Chung and D. Kim proved the following

TheOREM $1.2\left(\left[3\right.\right.$, Theorem 3.2]). Let $\alpha>1$. For any sequence $\left\{\mu_{n}\right\}_{n \in \mathbb{N}_{0}}$ of complex numbers there exists $f \in \mathcal{S}^{\alpha}(0, \infty)$ such that $\mu_{n}(f)=\mu_{n}$, $n \in \mathbb{N}_{0}$.

Our aim in this paper is to extend these results to the case of generalized Gelfand-Shilov spaces, $\mathcal{S}_{\boldsymbol{M}}(0, \infty)$ and $\mathcal{S}^{\boldsymbol{M}}(0, \infty)$, determined by means of a sequence $\boldsymbol{M}=\left\{M_{n}\right\}_{n \in \mathbb{N}_{0}}$ of positive numbers which will play the role of $n !^{\alpha-1}$ in the bounds in (1) and (2). In particular, previous work by the authors in the classical setting (see [12]) will be extended and improved.

Section 2 specifies the hypotheses on the sequence $\boldsymbol{M}$, needed for work with these spaces (and the corresponding subspaces of $\mathbb{C}^{\mathbb{N}_{0}}$ and related ultraholomorphic classes). We will make standard assumptions, such as logarithmic convexity, moderate growth and (strong) non-quasianalyticity. Following V. Thilliez [22], we define the growth index $\gamma(\boldsymbol{M})$ of a strongly regular sequence, crucial in what follows. In Section 3 we recall a theorem of 
V. Thilliez [22] which, under the condition $\gamma(\boldsymbol{M})>1$, proves the existence of linear continuous extension operators, right inverses for the Borel map, $f \mapsto\left\{f^{(n)}(0)\right\}_{n \in \mathbb{N}_{0}}$, for ultraholomorphic classes in sectors of the Riemann surface of the logarithm. This generalizes corresponding results in [19-21]. As our first statement, we prove that in some cases (specified by condition (5) below), including the Gevrey one, the condition $\gamma(\boldsymbol{M})>1$ is also necessary for such extensions to exist, or for the Borel map to be onto. Also, these classes turn out to be quasianalytic in wide enough sectors (in other words, a generalized Watson lemma is obtained).

In Section 4 we define general Gelfand-Shilov spaces, and study how the Fourier transform acts on them. Section 5 is devoted to the solution of the Stieltjes moment problem with general real exponents for the spaces $\mathcal{S}^{M}(0, \infty)$, where an idea of A. Yu. Popov [15] is fruitful.

Finally, Section 6 contains the results for $\mathcal{S}_{\boldsymbol{M}}(0, \infty)$. Here, the solution of the Stieltjes moment problem comes with linear continuous right inverses of the moment map, $f \mapsto\left\{\mu_{n}(f)\right\}_{n \in \mathbb{N}_{0}}$, in suitable subclasses. The proof rests on a careful analysis of the continuity of the different operations involved in the construction of the solution in [4], and it takes into account the extension operators for ultraholomorphic classes obtained by V. Thilliez [22]. Again, the necessity of the conditions imposed on $\boldsymbol{M}$ is studied, in such a way that, in some cases (which include $\mathcal{S}_{\alpha}(0, \infty)$ ), a complete answer is provided for our problem.

2. Strongly regular sequences. Let $\mathbb{N}=\{1,2, \ldots\}$ and $\mathbb{N}_{0}=\mathbb{N} \cup\{0\}$. In what follows, $\boldsymbol{M}=\left\{M_{n}\right\}_{n \in \mathbb{N}_{0}}$ will always stand for a sequence of positive real numbers, and we always assume that $M_{0}=1$.

Definition 2.1. Given a sequence $\boldsymbol{M}$ (as before), we say:

$\left(\alpha_{0}\right) \quad M$ is increasing if $M_{n} \leq M_{n+1}$ for every $n \in \mathbb{N}_{0}$.

$\left(\alpha_{1}\right) \boldsymbol{M}$ is logarithmically convex if $M_{n}^{2} \leq M_{n-1} M_{n+1}$ for every $n \in \mathbb{N}$.

( $\mu) \quad M$ is of moderate growth if there exists $A>0$ such that

$$
M_{n} \leq A^{n} \inf _{k, l \in \mathbb{N}_{0}, k+l=n} M_{k} M_{l}, \quad n \in \mathbb{N}_{0} .
$$

$(\gamma) \boldsymbol{M}$ satisfies the non-quasianalyticity condition if

$$
\sum_{n=0}^{\infty} \frac{M_{n}}{(n+1) M_{n+1}}<\infty .
$$

$\left(\gamma_{1}\right) \boldsymbol{M}$ satisfies the strong non-quasianalyticity condition if there exists $B>0$ such that

$$
\sum_{\ell \geq n} \frac{M_{\ell}}{(\ell+1) M_{\ell+1}} \leq B \frac{M_{n}}{M_{n+1}}, \quad n \in \mathbb{N}_{0} .
$$

It is obvious that $\left(\gamma_{1}\right) \Rightarrow(\gamma)$. 
Definition 2.2. A sequence $\boldsymbol{M}$ is said to be strongly regular if it has properties $\left(\alpha_{0}\right),\left(\alpha_{1}\right),(\mu)$ and $\left(\gamma_{1}\right)$.

REMARK 2.3. Of course, for a strongly regular sequence $\boldsymbol{M}$ the constants $A$ and $B$ above may be taken to be equal, and they cannot be less than 1 . It is easy to deduce that $\left(\alpha_{1}\right)$ and $(\gamma)$ together imply

$$
\lim _{n \rightarrow \infty} \frac{M_{n+1}}{M_{n}}=\lim _{n \rightarrow \infty} M_{n}^{1 / n}=\infty .
$$

We also note that we will mainly work with spaces of $\mathcal{C}^{\infty}$ or analytic functions defined by restricting the growth of their derivatives in terms of the sequence $\boldsymbol{N}:=\left\{n ! M_{n}\right\}_{n \in \mathbb{N}_{0}}$. However, some authors prefer to work directly in terms of the sequence $\boldsymbol{N}$, which leads to a reformulation of the conditions for strong regularity (see, for example, H. Komatsu [9] or J. Bruna [2]). In particular, if $\boldsymbol{M}$ is logarithmically convex, so is $\boldsymbol{N}$, but the converse does not necessarily hold.

Following [22], we next define the growth index of a strongly regular sequence.

Definition 2.4. Let $\gamma>0$. A strongly regular sequence $\boldsymbol{M}$ is said to have property $\left(P_{\gamma}\right)$ if there exist a sequence $p=\left\{p_{n}\right\}_{n \in \mathbb{N}_{0}}$ and a constant $a \geq 1$ such that:

(i) $a^{-1} M_{n+1} \leq M_{n} p_{n} \leq a M_{n+1}$ for all $n \in \mathbb{N}_{0}$,

(ii) $\left\{(n+1)^{-\gamma} p_{n}\right\}_{n \in \mathbb{N}_{0}}$ is increasing.

Proposition 2.5 ([22, Lemma 1.3.2]). Let $\boldsymbol{M}$ be a strongly regular sequence. Then:

(i) There exists $\gamma>0$ such that $\left(P_{\gamma}\right)$ holds, and there exists $a_{1}>0$ such that

$$
a_{1}^{n} n !^{\gamma} \leq M_{n}, \quad n \in \mathbb{N}_{0} .
$$

(ii) There exist $\delta>0$ and $a_{2}>0$ such that

$$
M_{n} \leq a_{2}^{n} n !^{\delta}, \quad n \in \mathbb{N}_{0} .
$$

Definition 2.6. The growth index of a strongly regular sequence $\boldsymbol{M}$ is defined as

$$
\gamma(\boldsymbol{M}):=\sup \left\{\gamma \in \mathbb{R}:\left(P_{\gamma}\right) \text { holds }\right\} .
$$

According to Proposition 2.5, we have $\gamma(\boldsymbol{M}) \in(0, \infty)$.

EXAMPLE 2.7. The most outstanding example of strongly regular sequences is that of Gevrey sequences, $\boldsymbol{M}=\left\{n !^{\alpha}\right\}_{n \in \mathbb{N}_{0}}$ for $\alpha>0$. It is plain to check that, in this case, $\gamma(\boldsymbol{M})=\alpha$. 
3. Extension results for ultraholomorphic classes. Now we introduce the ultraholomorphic classes we need. For convenience, given $d \in \mathbb{R}$ and $\theta>0$ we consider sectors in the Riemann surface $\Sigma$ of the logarithm with bisecting direction $d$ and opening $\theta \pi$,

$$
S_{d, \theta}=\{z \in \Sigma:|\arg z-d|<\theta \pi / 2\} .
$$

For a sequence $\boldsymbol{M}$ of positive real numbers and a constant $q \in \mathbb{N}$, we define the class $\mathcal{A}_{\boldsymbol{M}, q}\left(S_{d, \theta}\right)$ consisting of the holomorphic functions $f: S_{d, \theta} \rightarrow \mathbb{C}$ such that

$$
\|f\|_{M, q}:=\sup _{z \in S_{d, \theta}, n \in \mathbb{N}_{0}} \frac{\left|f^{(n)}(z)\right|}{q^{n} n ! M_{n}}<\infty,
$$

and the corresponding space of sequences of complex numbers,

$$
\Lambda_{M, q}:=\left\{\boldsymbol{\mu}=\left\{\mu_{n}\right\}_{n \in \mathbb{N}_{0}}:|\boldsymbol{\mu}|_{M, q}:=\sup _{n \in \mathbb{N}_{0}} \frac{\left|\mu_{n}\right|}{q^{n} n ! M_{n}}<\infty\right\} .
$$

$\left(\Lambda_{\boldsymbol{M}, q},||_{\boldsymbol{M}, q}\right)$ and $\left(\mathcal{A}_{\boldsymbol{M}, q}\left(S_{d, \theta}\right),\|\|_{\boldsymbol{M}, q}\right)$ are Banach spaces. Since the derivatives of an element $f \in \mathcal{A}_{M, q}\left(S_{d, \theta}\right)$ are Lipschitzian in $S_{d, \theta}$, they all extend continuously to $\bar{S}_{d, \theta}$, and in particular to 0 , and we put

$$
f^{(n)}(0):=\lim _{z \rightarrow 0, z \in S_{d, \theta}} f^{(n)}(z), \quad n \in \mathbb{N}_{0} .
$$

The Borel map $\mathcal{B}$, sending $f$ to $\left\{f^{(n)}(0)\right\}_{n \in \mathbb{N}_{0}}$, is then easily seen to be linear and continuous from $\mathcal{A}_{\boldsymbol{M}, q}\left(S_{d, \theta}\right)$ to $\Lambda_{\boldsymbol{M}, q}$. The next result, due to $\mathrm{V}$. Thilliez, generalizes the Borel-Ritt-Gevrey theorem $[16,17]$ by the construction of continuous right inverses for $\mathcal{B}$, and improves previous statements in this direction by the same author [21], J. Schmets and M. Valdivia [20], and J. Sanz [19].

Theorem 3.1 ([22, Theorem 3.2.1]). Let $\boldsymbol{M}$ be a strongly regular sequence, $d \in \mathbb{R}$, and $\theta$ be a real number with $0<\theta<\gamma(\boldsymbol{M})$. Then there exists $c \in \mathbb{N}$, depending only on $\boldsymbol{M}$ and $\theta$, such that for every $q \in \mathbb{N}$ there exists a linear continuous map

$$
U_{M, q, \theta}: \Lambda_{M, q} \rightarrow \mathcal{A}_{M, c q}\left(S_{d, \theta}\right)
$$

with $\mathcal{B} \circ U_{M, q, \theta}=\operatorname{Id}_{\Lambda_{M, q}}$.

REMARK 3.2. Our first result, coming next, shows that the condition $0<\theta<\gamma(\boldsymbol{M})$ is, under a simple assumption, indeed necessary for such extension operators to exist. The proof is an adaptation of the one supplied by J. Schmets and M. Valdivia for a similar statement regarding Gevrey classes [20, Theorem 5.11], which is thus generalized. 
Proposition 3.3. Let $M$ be a strongly regular sequence, $d \in \mathbb{R}$ and $\theta>0$, and suppose

$$
\sum_{n=0}^{\infty}\left(\frac{M_{n}}{M_{n+1}}\right)^{1 / \gamma(M)}=\infty .
$$

If there exists $q \in \mathbb{N}$ and a function $f \in \mathcal{A}_{\boldsymbol{M}, q}\left(S_{d, \theta}\right)$ such that $f^{(n)}(0)=\delta_{1, n}$ for every $n \in \mathbb{N}_{0}\left(\delta_{1, n}\right.$ is Dirac's delta $)$, then $\theta<\gamma(\boldsymbol{M})$.

Proof. Of course, it is enough to reason for $d=0$. Since $f$ is bounded on $S_{0, \theta}$, the function $\varphi: S_{0, \theta} \rightarrow \mathbb{C}, \varphi(z)=f(z)-z$, does not vanish identically in $S_{0, \theta}$. By Taylor's formula at 0 , there exists $A>0$ such that for every $z \in S_{0, \theta}$ with $|z| \leq 1$,

$$
|\varphi(z)| \leq A q^{n} M_{n}|z|^{n}, \quad n \in \mathbb{N}_{0} .
$$

Hence, the holomorphic function $\psi:\{z \in \mathbb{C}: \Re(z)>0\} \rightarrow \mathbb{C}$, defined by $\psi(u)=\varphi\left(1 / u^{\theta}\right)$, is not identically 0 and

$$
|\psi(u)| \leq \frac{A q^{n} M_{n}}{|u|^{\theta n}}, \quad n \in \mathbb{N}_{0}, \Re(u) \geq 1 .
$$

Now, we can apply Theorem 2.4.III of [13] and, taking into account (4), we deduce that

$$
\sum_{n=0}^{\infty}\left(\frac{M_{n}}{M_{n+1}}\right)^{1 / \theta}<\infty .
$$

But, according to (5), this would be impossible if we had $\theta \geq \gamma(\boldsymbol{M})$.

The previous proposition has two easy consequences. Firstly, if for a strongly regular sequence $\boldsymbol{M}$ we define

$$
\Lambda_{M}:=\bigcup_{q \in \mathbb{N}} \Lambda_{M, q}, \quad \mathcal{A}_{\boldsymbol{M}}\left(S_{d, \theta}\right):=\bigcup_{q \in \mathbb{N}} \mathcal{A}_{\boldsymbol{M}, q}\left(S_{d, \theta}\right),
$$

Theorem 3.1 tells us that the Borel map $\mathcal{B}: \mathcal{A}_{\boldsymbol{M}}\left(S_{d, \theta}\right) \rightarrow \Lambda_{\boldsymbol{M}}$ is onto whenever $\theta<\gamma(\boldsymbol{M})$. We have the following converse.

COROLlary 3.4. Let $\boldsymbol{M}$ be a strongly regular sequence satisfying (5), $d \in \mathbb{R}$ and $\theta \geq \gamma(\boldsymbol{M})$. Then the Borel map $\mathcal{B}: \mathcal{A}_{\boldsymbol{M}}\left(S_{d, \theta}\right) \rightarrow \Lambda_{\boldsymbol{M}}$ is not onto.

Secondly, since $\left\{\delta_{1, n}\right\}_{n \in \mathbb{N}_{0}} \in \Lambda_{M, q}$ for every $q \in \mathbb{N}$, we deduce the following.

COROLlaRY 3.5. Let $\boldsymbol{M}$ be a strongly regular sequence satisfying (5), $d \in \mathbb{R}$ and $\theta \geq \gamma(\boldsymbol{M})$. Then, given arbitrary $q, c \in \mathbb{N}$, there does not exist a linear continuous operator $U: \Lambda_{M, q} \rightarrow \mathcal{A}_{M, c q}\left(S_{d, \theta}\right)$ with $\mathcal{B} \circ U=\operatorname{Id}_{\Lambda_{M, q}}$. 
Example 3.6. For $\alpha>0$ and $\beta \in \mathbb{R}$, let us consider the sequence $\boldsymbol{P}=\left\{P_{n}\right\}_{n \geq 2}$ given by

$$
P_{n}=n !^{\alpha}\left(\prod_{k=2}^{n} \log (k)\right)^{\beta}, \quad n \geq 2 .
$$

It is not difficult to check that there exists $n_{0} \geq 2$ such that the sequence $\boldsymbol{M}=\left\{M_{n}\right\}_{n \in \mathbb{N}_{0}}$, where

$$
M_{n}=\frac{P_{n+n_{0}}}{P_{n_{0}}}, \quad n \in \mathbb{N}_{0},
$$

is strongly regular and $\gamma(\boldsymbol{M})=\alpha$. Moreover, it is plain to see that the condition (5) holds for $\boldsymbol{M}$ if, and only if, $\beta \leq \alpha$ (which, in particular, is true for Gevrey sequences, arising when $\beta=0$ ).

REMARK 3.7. Using a result of B. I. Korenblyum [10], V. Thilliez [22] states that the class $\mathcal{A}_{\boldsymbol{M}}\left(S_{d, \theta}\right)$ is quasianalytic (i.e., a function $f$ in the class such that $\mathcal{B}(f)=\mathbf{0}$ must identically vanish) if, and only if,

$$
\sum_{n=0}^{\infty}\left(\frac{M_{n}}{(n+1) M_{n+1}}\right)^{1 /(\theta+1)}=\infty .
$$

Thanks to [22, Lemma 2.2.1], he deduces that if $\theta<\gamma(\boldsymbol{M})$, then the class is non-quasianalytic. We point out that the converse also holds whenever the condition (5) is satisfied. Indeed, if the class is non-quasianalytic, it suffices to choose a function $f_{0}$ in this class, not identically 0 and with $\mathcal{B}\left(f_{0}\right)=\mathbf{0}$, and then repeat the proof of Proposition 3.3 starting from the consideration of the function $\varphi$, which must be set equal to $f_{0}$.

In particular, we have obtained the following result, which, according to the previous example, extends the classical Watson lemma, valid for Gevrey classes.

Proposition 3.8 (Generalized Watson lemma). Let $\boldsymbol{M}$ be a strongly regular sequence satisfying (5), and $\theta \geq \gamma(\boldsymbol{M})$. Then $\mathcal{A}_{\boldsymbol{M}}\left(S_{d, \theta}\right)$ is quasianalytic, or, in other words, (6) holds.

4. General Gelfand-Shilov spaces. We begin by defining some subspaces of the Schwartz space $\mathcal{S}(\mathbb{R})$ of rapidly decreasing smooth functions. Let $M$ be a sequence of positive real numbers, and $q \in \mathbb{N}$.

Definition 4.1. The space $\mathcal{S}_{M, q}$ consists of those $f \in \mathcal{S}(\mathbb{R})$ such that for each $n \in \mathbb{N}_{0}$,

$$
\sup _{x \in \mathbb{R}, m \in \mathbb{N}_{0}} \frac{\left|x^{m} f^{(n)}(x)\right|}{q^{m} m ! M_{m}}<\infty .
$$

We set $\mathcal{S}_{\boldsymbol{M}}:=\bigcup_{q \in \mathbb{N}} \mathcal{S}_{\boldsymbol{M}, q}$. 
$\mathcal{S}_{M, q}$ becomes a Fréchet space when endowed with the topology generated by the family $\left(p_{n}\right)_{n \in \mathbb{N}_{0}}$ of seminorms, defined as

$$
p_{n}(f)=\sup _{x \in \mathbb{R}, m \in \mathbb{N}_{0}} \frac{\left|x^{m} f^{(n)}(x)\right|}{q^{m} m ! M_{m}}, \quad f \in \mathcal{S}_{\boldsymbol{M}, q} .
$$

Of course, $\mathcal{S}_{\boldsymbol{M}, q}(0, \infty):=\left\{f \in \mathcal{S}_{\boldsymbol{M}, q}: \operatorname{supp}(f) \subset[0, \infty)\right\}$ is also a Fréchet space.

Definition 4.2. The space $\mathcal{S}^{M, q}$ consists of the functions $f \in \mathcal{S}(\mathbb{R})$ such that for each $m \in \mathbb{N}_{0}$,

$$
\sup _{x \in \mathbb{R}, n \in \mathbb{N}_{0}} \frac{\left|x^{m} f^{(n)}(x)\right|}{q^{n} n ! M_{n}}<\infty .
$$

We put $\mathcal{S}^{M}:=\bigcup_{q>0} \mathcal{S}^{M, q}$.

$\mathcal{S}^{M, q}$ is again a Fréchet space with the topology generated by the family $\left(p^{m}\right)_{m \in \mathbb{N}_{0}}$, where

$$
p^{m}(f)=\sup _{x \in \mathbb{R}, n \in \mathbb{N}_{0}} \frac{\left|x^{m} f^{(n)}(x)\right|}{q^{n} n ! M_{n}} .
$$

The space $\mathcal{S}^{\boldsymbol{M}, q}(0, \infty)$ is defined accordingly.

Remark 4.3. The spaces $\mathcal{S}_{\boldsymbol{M}}$ and $\mathcal{S}^{M}$ are named (general) GelfandShilov spaces [8] (although our notation slightly differs from the original one). In case $\boldsymbol{M}=\left\{n !^{\mid \alpha-1}\right\}_{n \in \mathbb{N}_{0}}$ with $\alpha>1$, we obtain the classical GelfandShilov spaces, usually denoted by $\mathcal{S}_{\alpha}$ and $\mathcal{S}^{\alpha}$, respectively.

The following results, needed later, describe how the Fourier transform

$$
\hat{f}(w)=\mathcal{F}(f)(w)=\int_{-\infty}^{\infty} f(t) e^{-i w t} d t, \quad w \in \mathbb{R},
$$

and its inverse

$$
\mathcal{F}^{-1}(g)(x)=\frac{1}{2 \pi} \int_{-\infty}^{\infty} g(w) e^{i w x} d w=\frac{1}{2 \pi} \mathcal{F}(g)(-x),
$$

act on the subspaces of $\mathcal{S}(\mathbb{R})$ we have just introduced. Although a proof of Propositions 4.4 and 4.5 can be obtained by suitably modifying the arguments in [8, Section IV.6], for the convenience of the reader we will sketch them. In each of them, we impose the simplest hypotheses on $\boldsymbol{M}$, all satisfied by a strongly regular sequence.

Proposition 4.4. Let $M$ be an increasing sequence of positive real numbers, and $q \in \mathbb{N}$. Then $\mathcal{F}\left(\mathcal{S}^{M, q}\right) \subseteq \mathcal{S}_{M, q}, \mathcal{F}^{-1}\left(\mathcal{S}^{M, q}\right) \subseteq \mathcal{S}_{\boldsymbol{M}, q}$, and the maps

$$
\mathcal{F}, \mathcal{F}^{-1}: \mathcal{S}^{M, q} \rightarrow \mathcal{S}_{M, q}
$$

are linear and continuous. 
Proof. In view of (7), it suffices to prove the assertion for $\mathcal{F}$. Let $\phi \in \mathcal{S}^{M, q}, w \in \mathbb{R}$, and $m, n \in \mathbb{N}_{0}$ be given. By differentiating under the integral sign and applying integration by parts $m$ times, we see that

$$
\begin{aligned}
\left|w^{m} \hat{\phi}^{(n)}(w)\right|= & \left|\int_{-\infty}^{\infty}\left(t^{n} \phi(t)\right)^{(m)} e^{-i w t} d t\right| \\
\leq & \sum_{k=0}^{\min \{m, n\}}\left(\begin{array}{c}
m \\
k
\end{array}\right) \frac{n !}{(n-k) !}\left[\int_{-1}^{1}\left|t^{n-k} \phi^{(m-k)}(t)\right| d t\right. \\
& \left.+\int_{1}^{\infty}\left|t^{n+2-k} \phi^{(m-k)}(t)\right| \frac{d t}{t^{2}}+\int_{-\infty}^{-1}\left|t^{n+2-k} \phi^{(m-k)}(t)\right| \frac{d t}{t^{2}}\right] .
\end{aligned}
$$

Since

$$
\left|t^{\nu} \phi^{(m-k)}(t)\right| \leq q^{m-k}(m-k) ! M_{m-k} p^{\nu}(\phi), \quad t \in \mathbb{R}, m, k, \nu \in \mathbb{N}_{0}, k \leq m,
$$

we deduce that

$$
\left|w^{m} \hat{\phi}^{(n)}(w)\right| \leq \sum_{k=0}^{\min \{m, n\}}\left(\begin{array}{c}
m \\
k
\end{array}\right) \frac{2 n ! q^{m-k}(m-k) ! M_{m-k}}{(n-k) !}\left(p^{n-k}(\phi)+p^{n+2-k}(\phi)\right) .
$$

Hence, as $\boldsymbol{M}$ is increasing,

$$
\begin{aligned}
\sup _{w \in \mathbb{R}, m \in \mathbb{N}_{0}} \frac{\left|w^{m} \hat{\phi}^{(n)}(w)\right|}{q^{m} m ! M_{m}} & \leq \sup _{m \in \mathbb{N}_{0}} \sum_{k=0}^{\min \{m, n\}}\left(\begin{array}{l}
n \\
k
\end{array}\right) \frac{M_{m-k}}{M_{m}} \frac{2}{q^{k}}\left(p^{n-k}(\phi)+p^{n+2-k}(\phi)\right) \\
& \leq \sum_{k=0}^{n}\left(\begin{array}{l}
n \\
k
\end{array}\right) \frac{2}{q^{k}}\left(p^{n-k}(\phi)+p^{n+2-k}(\phi)\right)<\infty,
\end{aligned}
$$

and we conclude that $\hat{\phi} \in \mathcal{S}_{M, q}$ and $\mathcal{F}: \mathcal{S}^{M, q} \rightarrow \mathcal{S}_{M, q}$ is continuous.

Proposition 4.5. Let $\boldsymbol{M}$ be an increasing sequence of moderate growth. Then there exists $c \in \mathbb{N}$ (depending on $\boldsymbol{M}$ ) such that for every $q \in \mathbb{N}$ we have $\mathcal{F}\left(\mathcal{S}_{M, q}\right) \subseteq \mathcal{S}^{M, c q}, \mathcal{F}^{-1}\left(\mathcal{S}_{\boldsymbol{M}, q}\right) \subseteq \mathcal{S}^{M, c q}$, and the maps

$$
\mathcal{F}, \mathcal{F}^{-1}: \mathcal{S}_{M, q} \rightarrow \mathcal{S}^{M, c q}
$$

are linear and continuous.

Proof. It is again enough to work with $\mathcal{F}$. Given $\phi \in \mathcal{S}_{M, q}$, we have

$$
\left|t^{\nu} \phi^{(m-k)}(t)\right| \leq q^{\nu} \nu ! M_{\nu} p_{m-k}(\phi), \quad t \in \mathbb{R}, \nu, k, m \in \mathbb{N}_{0}, k \leq m,
$$

Consequently, if one starts from the inequality (8), for $w \in \mathbb{R}$ and $m, n \in \mathbb{N}_{0}$ 
one gets

$$
\begin{aligned}
\left|w^{m} \hat{\phi}^{(n)}(w)\right| \leq & \sum_{k=0}^{\min \{m, n\}}\left(\begin{array}{c}
m \\
k
\end{array}\right) \frac{2 p_{m-k}(\phi) n !}{(n-k) !} \\
& \times\left[q^{n-k}(n-k) ! M_{n-k}+q^{n-k+2}(n-k+2) ! M_{n-k+2}\right] .
\end{aligned}
$$

Then, for a constant $c \in \mathbb{N}$, with $c>1$, to be determined, one can write

$$
\sup _{w \in \mathbb{R}, n \in \mathbb{N}_{0}} \frac{\left|w^{m} \hat{\phi}^{(n)}(w)\right|}{(c q)^{n} n ! M_{n}} \leq \sup _{n \in \mathbb{N}_{0}} \sum_{k=0}^{\min \{m, n\}} 2\left(\begin{array}{c}
m \\
k
\end{array}\right) p_{m-k}(\phi)\left[\frac{M_{n-k}}{M_{n} c^{n} q^{k}}+\eta_{k, n}\right]
$$

where

$$
\eta_{k, n}=\frac{M_{n-k+2}(n-k+2) !}{c^{n} q^{k-2} M_{n}(n-k) !} .
$$

On the one hand, as $\boldsymbol{M}$ is increasing and $c>1$, it is clear that

$$
\frac{M_{n-k}}{M_{n} c^{n} q^{k}} \leq \frac{1}{(c q)^{k}} \text {. }
$$

On the other hand, if $A \geq 1$ is the constant involved in the moderate growth of $M$ and we choose $c>A$, it is not difficult to check that

$$
\sup \left\{\eta_{k, n}: n \in \mathbb{N}_{0}, 0 \leq k \leq \min \{m, n\}\right\}=C<\infty .
$$

Hence, one finds

$$
\sup _{w \in \mathbb{R}, n \in \mathbb{N}_{0}} \frac{\left|w^{m} \hat{\phi}^{(n)}(w)\right|}{(c q)^{n} n ! M_{n}} \leq \sum_{k=0}^{m} 2\left(\begin{array}{c}
m \\
k
\end{array}\right) p_{m-k}(\phi)\left(\frac{1}{(c q)^{k}}+C\right),
$$

and the conclusions easily follow.

REMARK 4.6. The previous two propositions allow us to assert that, if $\boldsymbol{M}$ is an increasing sequence of moderate growth, then

$$
\mathcal{F}\left(\mathcal{S}_{M}\right)=\mathcal{S}^{M} \quad \text { and } \quad \mathcal{F}\left(\mathcal{S}^{M}\right)=\mathcal{S}_{\boldsymbol{M}}
$$

Another consequence of these results is the following characterization for the Fourier transforms of the elements of $\mathcal{S}_{\boldsymbol{M}}(0, \infty)$, generalizing a similar statement for classical Gelfand-Shilov spaces (see [4, Lemma 2.4]).

Proposition 4.7. Let $\boldsymbol{M}$ be an increasing sequence of moderate growth, $q \in \mathbb{N}$, and $\psi$ be a complex function defined in $\mathbb{R}$. The following statements are equivalent:

(i) $\psi$ is the Fourier transform of a function $\phi \in \mathcal{S}_{M, c_{1} q}(0, \infty)$ (for a certain $\left.c_{1} \in \mathbb{N}\right)$.

(ii) $\psi \in \mathcal{S}^{M, c_{2} q}$ (for a certain $c_{2} \in \mathbb{N}$ ), and $\psi$ may be extended to a function $\Psi$, continuous in $\bar{U}=\{z \in \mathbb{C}: \Im(z) \leq 0\}$, analytic in $U=$ $S_{-\pi / 2,1}$, and such that $\Psi \rightarrow 0$ as $z \rightarrow \infty$ in $\bar{U}$. 
5. Stieltjes moment problem in $\mathcal{S}^{M}(0, \infty)$. In this section we will generalize Theorem 1.2 in two respects: the exponents of $t$ in the integrals defining the moments may be a sequence more general than just $\{n\}_{n \in \mathbb{N}_{0}}$, and the function $f$ may be chosen in the space $\mathcal{S}^{M}(0, \infty)$, for a sequence $\boldsymbol{M}$ subject to some mild conditions. In the authors' opinion, it is not possible to obtain any of these two enhancements by following the same argument as in [3].

We will make use of the following result of Denjoy-Carleman-Mandelbrojt, whose proof may be found in [9, Theorem 4.2].

Lemma 5.1. Suppose $\boldsymbol{M}=\left\{M_{n}\right\}_{n \in \mathbb{N}_{0}}$ is a sequence which satisfies $(\gamma)$ and such that $\left\{n ! M_{n}\right\}_{n \in \mathbb{N}_{0}}$ satisfies $\left(\alpha_{1}\right)$. Then there exists a $\mathcal{C}^{\infty}$ function $\varphi: \mathbb{R} \rightarrow[0, \infty)$, not identically 0 , such that $\operatorname{supp}(\varphi) \subset[0,1]$, and such that there exists $q \in \mathbb{N}$ with

$$
\sup _{x \in[0,1], n \in \mathbb{N}_{0}} \frac{\left|\varphi^{(n)}(x)\right|}{q^{n} n ! M_{n}}<\infty .
$$

REMARK 5.2. By the very definition of the Gelfand-Shilov spaces, it is clear that the function $\varphi$ in the above lemma belongs to $\mathcal{S}^{M}(0, \infty)$.

Also, we note that for every $\alpha \in \mathbb{R}$ and every $f \in \mathcal{S}^{M}(0, \infty)$, the integral

$$
\int_{0}^{\infty} t^{\alpha} f(t) d t
$$

makes sense, since for every $\nu \in \mathbb{N}$ we have

$$
\lim _{t \rightarrow 0} \frac{f(t)}{t^{\nu}}=0, \quad \lim _{t \rightarrow \infty} t^{\nu} f(t)=0 .
$$

We proceed to the statement of our result, whose proof is based on an idea of A. Yu. Popov [15].

THEOREM 5.3. Let $\boldsymbol{M}=\left\{M_{n}\right\}_{n \in \mathbb{N}_{0}}$ be a sequence as in Lemma 5.1. Let $\left\{\alpha_{n}\right\}_{n \in \mathbb{N}_{0}}$ be a sequence of real numbers such that $\alpha_{m} \neq \alpha_{n}$ whenever $m \neq n$, and $\lim _{n \rightarrow \infty} \alpha_{n}=+\infty$. Then for any sequence $\left\{\mu_{n}\right\}_{n \in \mathbb{N}_{0}}$ of complex numbers there exists $f \in \mathcal{S}^{M}(0, \infty)$ such that

$$
\int_{0}^{\infty} t^{\alpha_{n}} f(t) d t=\mu_{n}, \quad n \in \mathbb{N}_{0} .
$$

Proof. We fix the function $\varphi$ and the natural number $q$ provided by the lemma, and put

$$
\varphi_{n}:=\int_{0}^{1} t^{\alpha_{n}} \varphi(t) d t>0, \quad n \in \mathbb{N}_{0} .
$$

We choose a real number $r>1$. Then $r^{\alpha_{n}} \neq r^{\alpha_{m}}$ for $n \neq m$, and $\lim _{n \rightarrow \infty} r^{\alpha_{n}}$ $=\infty$. So, by a standard consequence of the theorem of Mittag-Leffler 
(see [18, p. 134]), there exists an entire function $g(z)=\sum_{k=0}^{\infty} a_{k} z^{k}$ such that

$$
g\left(r^{\alpha_{n}}\right)=\mu_{n} / \varphi_{n}, \quad n \in \mathbb{N}_{0} .
$$

We set, at least formally,

$$
f(t)=\sum_{k=0}^{\infty} a_{k} r^{-k} \varphi\left(r^{-k} t\right), \quad t \in \mathbb{R} .
$$

Given $m, n \in \mathbb{N}_{0}$, on the one hand we know there exists $C=C(\varphi)>0$ such that

$$
\left|t^{m} \varphi^{(n)}(t)\right| \leq C q^{n} n ! M_{n}, \quad t \in \mathbb{R} .
$$

On the other hand, since $g$ is entire, $c_{m}:=\sum_{k=0}^{\infty}\left|a_{k}\right| r^{m k}<\infty$. Then we can estimate the product of $t^{m}$ times the $n$th formal derivative of the series in (9) as follows:

$$
\begin{aligned}
\left|t^{m} \sum_{k=0}^{\infty} a_{k} r^{-k}\left(r^{-k}\right)^{n} \varphi^{(n)}\left(r^{-k} t\right)\right| & \leq \sum_{k=0}^{\infty}\left|a_{k}\right| r^{(m-n-1) k}\left|\left(r^{-k} t\right)^{m} \varphi^{(n)}\left(r^{-k} t\right)\right| \\
& \leq C q^{n} n ! M_{n} \sum_{k=0}^{\infty}\left|a_{k}\right| r^{m k}=C c_{m} q^{n} n ! M_{n} .
\end{aligned}
$$

This estimate shows that $f$ is well defined and belongs to $\mathcal{S}^{M}(0, \infty)$; moreover, we can apply the Lebesgue convergence theorem to deduce that, for every $n \in \mathbb{N}_{0}$,

$$
\begin{aligned}
\int_{0}^{\infty} t^{\alpha_{n}} f(t) d t & =\sum_{k=0}^{\infty} a_{k} r^{-k} \int_{0}^{\infty} t^{\alpha_{n}} \varphi\left(r^{-k} t\right) d t \\
& =\sum_{k=0}^{\infty} a_{k} r^{\alpha_{n} k} \int_{0}^{\infty} u^{\alpha_{n}} \varphi(u) d u=g\left(r^{\alpha_{n}}\right) \varphi_{n}=\mu_{n},
\end{aligned}
$$

as desired.

6. Stieltjes moment problem in $\mathcal{S}_{M, q}(0, \infty)$. Regarding the moments of an element in $\mathcal{S}_{\boldsymbol{M}}$, we have the following result.

Proposition 6.1. Let $\boldsymbol{M}$ be a sequence of moderate growth. Then there exists $c \in \mathbb{N}, c>1$, such that whenever $f \in \mathcal{S}_{M, q}(0, \infty)$ for some $q \in \mathbb{N}$, one has $\mathcal{M}(f):=\left\{\mu_{n}(f)\right\}_{n \in \mathbb{N}_{0}} \in \Lambda_{\boldsymbol{M}, c q}$, and the moment map $\mathcal{M}$ : $\mathcal{S}_{M, q}(0, \infty) \rightarrow \Lambda_{M, c q}$ is continuous.

Proof. Let $A \geq 1$ be the constant appearing in (3), and fix $c \in \mathbb{N}$ with $c>A$. Choose $C>0$ such that

$$
A^{n}(n+2)(n+1) \leq C c^{n}, \quad n \in \mathbb{N}_{0} .
$$


For $q \in \mathbb{N}, f \in \mathcal{S}_{M, q}(0, \infty)$ and $n \in \mathbb{N}_{0}$ one has

$$
\begin{aligned}
\left|\mu_{n}(f)\right| & \leq \int_{0}^{1}\left|x^{n} f(x)\right| d x+\int_{1}^{\infty}\left|x^{n+2} f(x)\right| \frac{d x}{x^{2}} \\
& \leq q^{n} n ! M_{n} p_{0}(f)+q^{n+2}(n+2) ! M_{n+2} p_{0}(f) \\
& \leq q^{n} n ! M_{n} p_{0}(f)\left[1+q^{2}(n+2)(n+1) A^{n+2} M_{2}\right] \\
& \leq 2 q^{2} A^{2} M_{2} C p_{0}(f)(c q)^{n} n ! M_{n},
\end{aligned}
$$

so

$$
|\mathcal{M}(f)|_{M, c q}=\sup _{n \in \mathbb{N}_{0}} \frac{\left|\mu_{n}(f)\right|}{(c q)^{n} n ! M_{n}} \leq 2 q^{2} A^{2} M_{2} C p_{0}(f),
$$

from which the conclusion follows.

Our main goal in this section is to obtain a converse of the last statement, as follows.

THEOREM 6.2. Let $\boldsymbol{M}$ be a strongly regular sequence with $\gamma(\boldsymbol{M})>1$. Then there exists $c \in \mathbb{N}$ (depending on $\boldsymbol{M}$ ) such that for every $q \in \mathbb{N}$ there is a linear continuous map

$$
T_{M, q}: \Lambda_{M, q} \rightarrow \mathcal{S}_{M, c q}(0, \infty)
$$

such that $\mathcal{M} \circ T_{M, q}=\operatorname{Id}_{\Lambda_{M, q}}$.

This result generalizes a previous result by the authors, where only the spaces $\mathcal{S}_{\alpha}(0, \infty)$, with $\alpha>2$, were considered (see Remark 4.3).

REMARK 6.3. We briefly outline the proof in order to clarify the steps to follow. Given $\mu=\left\{\mu_{n}\right\}_{n \in \mathbb{N}_{0}} \in \Lambda_{M, q}$, we are searching for a function $T_{M, q}(\mu) \in \mathcal{S}_{M, c q}(0, \infty)$ (for some $c \in \mathbb{N}$ ) such that

$$
\int_{0}^{\infty} t^{n} \cdot T_{M, q}(\mu)(t) d t=\mu_{n}, \quad n \in \mathbb{N}_{0} .
$$

Hence, if we put $\psi=\mathcal{F}\left(T_{\boldsymbol{M}, q}(\mu)\right)$, we easily deduce that

$$
\psi^{(n)}(0)=(-i)^{n} \int_{0}^{\infty} t^{n} \cdot T_{M, q}(\mu)(t) d t=(-i)^{n} \mu_{n}, \quad n \in \mathbb{N}_{0} .
$$

We will build a function satisfying (10), and this will be done in such a way that its inverse Fourier transform belongs to $\mathcal{S}_{M, c q}(0, \infty)$. Indeed, $\psi$ will be the restriction to the real axis of the product of two other functions, both holomorphic in suitable sectors containing $\mathbb{R} \backslash\{0\}$. The first one will be an auxiliary function, say $G$, tending to 0 as $|z|$ tends to infinity, and the second one will be a function $F$, in some ultraholomorphic class $\mathcal{A}_{\boldsymbol{M}, \tilde{c} q}\left(S_{-\pi / 2, \theta}\right)$ (where $\tilde{c} \in \mathbb{N}$ and $\theta>1$ ), whose derivatives at 0 are determined according 
to Leibniz's rule in order to satisfy the equality of the first and last terms in (10): indeed, if $(1 / G)^{(n)}(0)=a_{n}, n \in \mathbb{N}_{0}$, one must require

$$
F^{(n)}(0)=\sum_{k=0}^{n}\left(\begin{array}{l}
n \\
k
\end{array}\right)(-i)^{k} \mu_{k} a_{n-k}, \quad n \in \mathbb{N}_{0} .
$$

Since this last sequence $\left\{F^{(n)}(0)\right\}_{n \in \mathbb{N}_{0}}$ turns out to belong to $\Lambda_{\boldsymbol{M}, c_{1} q}$ for suitable $c_{1} \in \mathbb{N}$ (see Proposition 6.4), such an $F$ will be obtained thanks to the operators $U_{M, q, \theta}$ in Theorem 3.1.

We next describe the auxiliary function to be used later on. For $\tau>1$ and $H=\mathbb{C}-\{i y: y \geq 1\}$ we define the function

$$
h_{\tau}(z)=e^{\pi i(1+1 /(2 \tau))}(z-i)^{1 / \tau}, \quad z \in H,
$$

where the determination of the logarithm is specified by

$$
\arg (z-i) \in(-3 \pi / 2, \pi / 2) .
$$

The function $G_{\tau}: H \rightarrow \mathbb{C}$ given by

$$
G_{\tau}(z)=\exp \left(h_{\tau}(z)\right), \quad z \in H,
$$

is holomorphic in $H$ and does not vanish, so that $1 / G_{\tau}$ is analytic in $H$ (and, in particular, at 0 ). The relevant feature (see [12, Lemma 4.10]) is that, if $\varepsilon$ is such that $0<\varepsilon<\min \{\pi(\tau-1) / 2, \pi / 2\}$, then $G_{\tau}(z)$ tends to 0 as $z$ tends to $\infty$ in the sector

$$
V_{\varepsilon}=\{z \in H: \arg (z-i) \in(-\pi-\varepsilon, \varepsilon)\} .
$$

The following result regards the map, defined between sequence spaces, given by the relations in (11).

Proposition 6.4. Let $\boldsymbol{M}$ be an increasing sequence, and let $\left\{a_{n}\right\}_{n \in \mathbb{N}_{0}}$ be the sequence of derivatives at 0 of a function $g$, holomorphic at that point. Then there exists $c \in \mathbb{N}$ such that for every $q \in \mathbb{N}$, the map

$$
\begin{gathered}
T_{1}: \Lambda_{\boldsymbol{M}, q} \rightarrow \Lambda_{\boldsymbol{M}, c q}, \\
\boldsymbol{\mu}=\left\{\mu_{n}\right\}_{n \in \mathbb{N}_{0}} \mapsto \boldsymbol{b}=\left\{b_{n}\right\}_{n \in \mathbb{N}_{0}}, \quad b_{n}=\sum_{k=0}^{n}\left(\begin{array}{l}
n \\
k
\end{array}\right)(-i)^{k} \mu_{k} a_{n-k},
\end{gathered}
$$

is well defined, linear and continuous.

Proof. Let $\varrho \in(0, \infty]$ be the radius of convergence of the Taylor series of $g$ at 0 , and choose $c \in \mathbb{N}$ such that $1 /(c q)<\varrho$ if $\varrho$ is finite; otherwise, $c$ may be any integer greater than 1 . Since $M$ is increasing, for every $n \in \mathbb{N}_{0}$ 
we have

$$
\begin{aligned}
\frac{\left|b_{n}\right|}{(c q)^{n} n ! M_{n}} & \leq \sum_{k=0}^{n}\left(\begin{array}{l}
n \\
k
\end{array}\right) \frac{\left.|\mu|\right|_{M, q} q^{k} k ! M_{k}\left|a_{n-k}\right|}{c^{n-k} q^{n} n ! M_{n}} \\
& \leq|\mu|_{M, q} \sum_{k=0}^{n} \frac{\left|a_{n-k}\right|}{(n-k) !(c q)^{n-k}} \leq|\mu|_{M, q} \sum_{k=0}^{\infty} \frac{\left|a_{k}\right|}{k !(c q)^{k}}<\infty .
\end{aligned}
$$

Hence $b \in \Gamma_{M, c q}$, and the continuity of $T_{1}$ follows.

Next we deal, firstly, with the product of an element in some class $\mathcal{A}_{M, q}\left(S_{-\pi / 2, \theta}\right)$ and a holomorphic function bounded at infinity, and secondly, with its restriction to the real axis when this bounded function is $G_{\tau}$. Similar results were proven in $[12,4.11$ and 4.13$]$ for the case $M=\left\{n !^{\alpha-1}\right\}_{n \in \mathbb{N}_{0}}$, with $\alpha>1$. Since the arguments are essentially the same, we just state the following two results under the least restrictive assumptions on the sequence $\boldsymbol{M}$.

Lemma 6.5. Let $M$ be an increasing sequence. Suppose $\theta>1$ and $\varepsilon>0$ are such that the sector $V_{\varepsilon}$, defined in (13), contains the sector $S_{-\pi / 2, \theta}$, and suppose $G$ is a holomorphic function in $V_{\varepsilon}$ such that for suitable $M, R>0$ we have

$$
|G(z)| \leq M \quad \text { for all } z \in V_{\varepsilon} \text { with }|z| \geq R \text {. }
$$

Then there exists $c \in \mathbb{N}$ such that for every $q \in \mathbb{N}$ and $f \in \mathcal{A}_{M, q}\left(S_{-\pi / 2, \theta}\right)$ one has $f G \in \mathcal{A}_{M, c q}\left(S_{-\pi / 2, \theta}\right)$.

Proposition 6.6. Let $\boldsymbol{M}, \theta>1$ and $\varepsilon>0$ be as in the previous result. For $\tau>1$, let $G_{\tau}: V_{\varepsilon} \rightarrow \mathbb{C}$ be the function defined in (12). Then there exists $c \in \mathbb{N}$ such that for every $q \in \mathbb{N}$ :

(i) For every $F \in \mathcal{A}_{\boldsymbol{M}, q}\left(S_{-\pi / 2, \theta}\right)$ one has $\left.F G_{\tau}\right|_{\mathbb{R}} \in \mathcal{S}^{M, c q}$.

(ii) The map

$$
T_{2}: \mathcal{A}_{M, q}\left(S_{-\pi / 2, \theta}\right) \rightarrow \mathcal{S}^{M, c q}, \quad F \rightarrow T_{2} F=\left.\left(F G_{\tau}\right)\right|_{\mathbb{R}},
$$

is linear and continuous.

REMARK 6.7. In the conditions of the previous result, $U=S_{-\pi / 2,1}$ satisfies $\bar{U} \subseteq \bar{S}_{-\pi / 2, \theta}$. So, given $F \in \mathcal{A}_{M, q}\left(S_{-\pi / 2, \theta}\right)$, the function $\left.\left(F G_{\tau}\right)\right|_{\bar{U}}$ is continuous in $\bar{U}$, analytic in $U$, extends $T_{2} F$, and

$$
\left.\lim _{z \rightarrow \infty, z \in \bar{U}}\left(F G_{\tau}\right)\right|_{\bar{U}}(z)=0 .
$$

In view of Proposition 4.7, $T_{2} F$ is the Fourier transform of a function in $\mathcal{S}_{M, c q}(0, \infty)$, with suitable $c \in \mathbb{N}$.

Finally, we end up with the proof of our main result. 
Proof of Theorem 6.2. Fix $\tau>1$, and let $a_{n}:=\left(1 / G_{\tau}\right)^{(n)}(0), n \in \mathbb{N}_{0}$. Proposition 6.4 gives us $c_{1} \in \mathbb{N}$ and the linear continuous map $T_{1}$ defined by means of $\left\{a_{n}\right\}_{n \in \mathbb{N}_{0}}$. Choose $\theta \in(1, \gamma(\boldsymbol{M}))$. According to Theorem 3.1, there exist $c_{2} \in \mathbb{N}$ and a linear continuous map $U_{\boldsymbol{M}, c_{1} q, \theta}$ such that $\mathcal{B} \circ U_{M, c_{1} q, \theta}=\operatorname{Id}_{\Lambda_{M, c_{1} q}}$. Then Proposition 6.6 gives a constant $c_{3} \in \mathbb{N}$ and a linear continuous map

$$
T_{2}: \mathcal{A}_{M, c_{2} c_{1} q}\left(S_{-\pi / 2, \theta}\right) \rightarrow \mathcal{S}^{M, c_{3} c_{2} c_{1} q} .
$$

By Proposition 4.4 we know that $\mathcal{F}^{-1}$ sends $\mathcal{S}^{M, c_{3} c_{2} c_{1} q}$ into $\mathcal{S}_{\boldsymbol{M}, c_{3} c_{2} c_{1} q}$ continuously. Setting $c=c_{3} c_{2} c_{1} \in \mathbb{N}$, it is easy to check (see Remarks 6.3 and 6.7) that

$$
T_{M, q}:=\mathcal{F}^{-1} \circ T_{2} \circ U_{M, c_{1} q, \theta} \circ T_{1}: \Lambda_{\boldsymbol{M}, q} \rightarrow \mathcal{S}_{\boldsymbol{M}, c q}(0, \infty)
$$

solves the problem.

We now turn our attention to the necessity of the conditions in Theorem 6.2.

Proposition 6.8. Let $\boldsymbol{M}$ be a strongly regular sequence satisfying (5). If there exists $q \in \mathbb{N}$ and a function $f \in \mathcal{S}_{\boldsymbol{M}, q}(0, \infty)$ such that $\mu_{n}(f)=$ $(-1)^{n} \delta_{1, n}$ for every $n \in \mathbb{N}_{0}$, then $\gamma(\boldsymbol{M})>1$.

Proof. We consider the Laplace transform of $f$,

$$
g(z)=\int_{0}^{\infty} e^{-z t} f(t) d t .
$$

The function $g$ is clearly defined for $\Re(z) \geq 0$, where it is continuous, and it is holomorphic in $H=\{z \in \mathbb{C}: \Re(z)>0\}$. Moreover, since for any $n \in \mathbb{N}_{0}$ we have

$$
\left|t^{n} f(t)\right| \leq p_{0}(f) q^{n} n ! M_{n}, \quad t \in(0, \infty),
$$

for every $z$ with $\Re(z) \geq 0$ we deduce that

$$
\begin{aligned}
\left|g^{(n)}(z)\right| & \leq \int_{0}^{\infty}\left|(-1)^{n} e^{-z t} t^{n} f(t)\right| d t \leq \int_{0}^{1}\left|t^{n} f(t)\right| d t+\int_{1}^{\infty}\left|t^{n+2} f(t)\right| \frac{d t}{t^{2}} \\
& \leq p_{0}(f) q^{n} n ! M_{n}+p_{0}(f) q^{n+2}(n+2) ! M_{n+2} .
\end{aligned}
$$

As in the proof of Proposition 6.1, we see there exists $c \in \mathbb{N}, c>1$, such that $g \in \mathcal{A}_{\boldsymbol{M}, c q}\left(S_{0,1}\right)$. But we also have

$$
g^{(n)}(0)=\int_{0}^{\infty}(-1)^{n} t^{n} f(t) d t=(-1)^{n} \mu_{n}(f)=\delta_{1, n}, \quad n \in \mathbb{N}_{0} .
$$

Then Proposition 3.3 ensures that $1<\gamma(\boldsymbol{M})$.

For a strongly regular sequence $\boldsymbol{M}$ with $\gamma(\boldsymbol{M})>1$, Theorem 6.2 allows us to assert that $\mathcal{M}: \mathcal{S}_{\boldsymbol{M}}(0, \infty) \rightarrow \Lambda_{\boldsymbol{M}}$ is onto. Conversely, we have 
COROLlary 6.9. Let $\boldsymbol{M}$ be a strongly regular sequence satisfying (5) and with $\gamma(\boldsymbol{M}) \leq 1$. Then:

(i) The map $\mathcal{M}: \mathcal{S}_{\boldsymbol{M}}(0, \infty) \rightarrow \Lambda_{M}$ is not onto.

(ii) For arbitrary $q, c \in \mathbb{N}$, there does not exist a linear continuous operator

$$
T: \Lambda_{M, q} \rightarrow \mathcal{S}_{M, c q}(0, \infty)
$$

with $\mathcal{M} \circ T=\operatorname{Id}_{\Lambda_{M, q}}$.

REMARK 6.10. The previous results give a complete solution of the Stieltjes moment problem in the general Gelfand-Shilov spaces $\mathcal{S}_{M}(0, \infty)$ under condition (5). This includes the case of the classical spaces $\mathcal{S}_{\alpha}(0, \infty)$.

Finally, we obtain a result on the necessity of condition $\left(\gamma_{1}\right)$.

Proposition 6.11. Suppose $\boldsymbol{M}=\left\{M_{n}\right\}_{n \in \mathbb{N}_{0}}$ is a sequence which satisfies $(\gamma)$ and such that $\left\{n ! M_{n}\right\}_{n \in \mathbb{N}_{0}}$ satisfies $\left(\alpha_{1}\right)$. If there exist $q, c \in \mathbb{N}$ and a linear continuous operator $T: \Lambda_{M, q} \rightarrow \mathcal{S}_{M, c q}(0, \infty)$ such that $\mathcal{M} \circ T=$ $\operatorname{Id}_{\Lambda_{M, q}}$, then the sequence $\boldsymbol{M}$ satisfies $\left(\gamma_{1}\right)$.

Proof. The map $\left\{\mu_{n}\right\}_{n \in \mathbb{N}_{0}} \rightarrow\left\{\mu_{n} /(-i)^{n}\right\}_{n \in \mathbb{N}_{0}}$ is an isomorphism, say $\rho$, in $\Lambda_{M, q}$. For $r \in \mathbb{N}$, consider the space $\mathcal{E}_{M, r}[-1,1]$ consisting of the functions $f \in \mathcal{C}^{\infty}[-1,1]$ such that $|f|_{[-1,1], r}<\infty$, where

$$
|f|_{[-1,1], r}=\sup _{n \in \mathbb{N}_{0}, x \in[-1,1]} \frac{\left|f^{(n)}(x)\right|}{r^{n} n ! M_{n}} .
$$

$\left(\mathcal{E}_{M, r}[-1,1],||_{[-1,1], r}\right)$ is a Banach space, and the restriction map $j: \mathcal{S}^{M, r} \rightarrow$ $\mathcal{E}_{M, r}[-1,1]$ is well-defined, linear and continuous, since

$$
|f|_{[-1,1], r}=\sup _{x \in[-1,1], n \in \mathbb{N}} \frac{\left|f^{(n)}(x)\right|}{r^{n} n ! M_{n}} \leq p^{0}(f), \quad f \in \mathcal{S}^{M, r} .
$$

Now, applying Proposition 4.5, it is easy to check that there exists $c_{1} \in \mathbb{N}$ such that the map $T_{1}:=j \circ \mathcal{F} \circ T \circ \rho$ is linear continuous from $\Lambda_{M, q}$ to $\mathcal{E}_{M, c_{1} q}[-1,1]$ and satisfies $\mathcal{B} \circ T_{1}=\operatorname{Id}_{\Lambda_{M, q}}$. It suffices to take into account Theorem 3.6 in the paper of H.-J. Petzsche [14] to conclude.

Acknowledgements. The first author is supported by Consejería de Educación y Cultura (Junta de Castilla y León) and Fondo Social Europeo.

The second author is partly supported by Consejería de Educación y Cultura (Junta de Castilla y León) under project VA103A05.

\section{References}

[1] N. I. Akhiezer, The Classical Moment Problem, Hafner, New York, 1965.

[2] J. Bruna, An extension theorem of Whitney type for non-quasi-analytic classes of functions, J. London Math. Soc. (2) 22 (1980), 495-505. 
[3] J. Chung, S.-Y. Chung and D. Kim, Every Stieltjes moment problem has a solution in Gelfand-Shilov spaces, J. Math. Soc. Japan 55 (2003), 909-913.

[4] S.-Y. Chung, D. Kim and Y. Yeom, Stieltjes moment problem for Gel'fand-Leontiev spaces, Fract. Calc. Appl. Anal. 2 (1999), 623-629.

[5] A. J. Durán, The Stieltjes moments problem for rapidly decreasing functions, Proc. Amer. Math. Soc. 107 (1989), 731-741.

[6] A. L. Durán and R. Estrada, Strong moment problems for rapidly decreasing smooth functions, ibid. 120 (1994), 529-534.

[7] R. Estrada and R. P. Kanwal, A Distributional Approach to Asymptotics. Theory and Applications, 2nd ed., Birkhäuser, Boston, MA, 2002.

[8] I. M. Gelfand and G. E. Shilov, Generalized Functions, Vol. 2, Spaces of Fundamental and Generalized Functions, Academic Press, New York, 1968.

[9] H. Komatsu, Ultradistributions, I: Structure theorems and a characterization, J. Fac. Sci. Univ. Tokyo Sect. IA Math. 20 (1973), 25-105.

[10] V. I. Korenblyum, Conditions of nontriviality of certain classes of functions analytic in a sector, and problems of quasianalyticity, Soviet Math. Dokl. 7 (1966), 232-236.

[11] H. J. Landau (ed.), Moments in Mathematics, Proc. Sympos. Appl. Math. 37, Amer. Math. Soc., Providence, RI, 1987.

[12] A. Lastra and J. Sanz, Linear continuous operators for the Stieltjes moment problem in Gelfand-Shilov spaces, J. Math. Anal. Appl. 340 (2008), 968-981.

[13] S. Mandelbrojt, Séries adhérentes. Régularisation des suites. Applications, Gauthier-Villars, Paris, 1952.

[14] H.-J. Petzsche, On E. Borel's theorem, Math. Ann. 282 (1988), 299-313.

[15] A. Yu. Popov, The moments problem for rapidly decreasing functions, Math. Notes 60 (1996), 49-55.

[16] J. P. Ramis, Dévissage Gevrey, Astérisque 59-60 (1978), 173-204.

[17] —, Les séries k-sommables et leurs applications, Lecture Notes in Phys. 126, Springer, Berlin, 1980.

[18] R. Remmert, Classical Topics in Complex Function Theory, Springer, New York, 1998.

[19] J. Sanz, Linear continuous extension operators for Gevrey classes on polysectors, Glasgow Math. J. 45 (2003), 199-216.

[20] J. Schmets and M. Valdivia, Extension maps in ultradifferentiable and ultraholomorphic function spaces, Studia Math. 143 (2000), 221-250.

[21] V. Thilliez, Extension Gevrey et rigidité dans un secteur, ibid. 117 (1995), 29-41.

[22] —, Division by flat ultradifferentiable functions and sectorial extensions, Results Math. 44 (2003), 169-188.

Departamento de Análisis Matemático y Didáctica de la Matemática

Facultad de Ciencias

Universidad de Valladolid

Paseo del Prado de la Magdalena s/n

47005 Valladolid, Spain

E-mail: jsanzg@am.uva.es

Received April 3, 2008

Revised version October 21, 2008 